\title{
ELEMENTARY EQUTVALENCE FOR FINITELY GENERATED NILPOTENT GROUPS AND MULTILINEAR MAPS
}

\section{Francis Oger}

We show that two finitely generated finite-by-nilpotent groups are elementarily equivalent if and only if they satisfy the same sentences with two alternations of quantifiers. For each integer $n \geqslant 2$, we prove the same result for the following classes of structures:

(1) the $(n+2)$-tuples $\left(A_{1}, \ldots, A_{n+1}, f\right)$, where $A_{1}, \ldots, A_{n+1}$ are disjoint finitely generated Abelian groups and $f: A_{1} \times \cdots \times A_{n} \rightarrow A_{n+1}$ is a $n$-linear map;

(2) the triples $(A, B, f)$, where $A, B$ are disjoint finitely generated Abelian groups and $f: A^{n} \rightarrow B$ is a $n$-linear map;

(3) the pairs $(A, f)$, where $A$ is a finitely generated Abelian group and $f: A^{n} \rightarrow A$ is a $n$-linear map.

In the proof, we use some properties of commutative rings associated to multilinear maps.

It is well known that two modules, and in particular two Abelian groups, are elementarily equivalent if and only if they satisfy the same $\forall \exists$ sentences (see [14, Corollary 2.18 , p.37]). In [13], we showed that two Abelian-by-finite groups are elementarily equivalent if and only if they satisfy the same $\exists \forall \exists$ sentences.

For non Abelian-by-finite groups, the situation is radically different. Burris proved in [1] that, for each integer $n$, there exist two groups which satisfy the same sentences with $n$ alternations of quantifiers without being elementarily equivalent. The groups in Burris' example are soluble since they are in the variety generated by the symmetric group on 3 letters $S_{3}$.

Moreover, we showed in [13] that, for each integer $n$, there exist two nilpotent groups which satisfy the same sentences with $n$ alternations of quantifiers and do not satisfy the same sentences with $n+1$ alternations of quantifiers.

In contrast with this result, we prove in the present paper that two finitely generated finite-by-nilpotent groups are elementarily equivalent if and only if they satisfy the same $\exists \forall \exists$ sentences.

On the other hand, the following questions are currently open:

Received 20th April, 1998

We express our thanks to A.G. Myasnikov for helpful conversations concerning [4], [5] and [6], during his visit in Paris, in 1993.

Copyright Clearance Centre, Inc. Serial-fee code: 0004-9729/98 \$A2.00+0.00. 
(1) Is there an integer $n$ such that two finitely generated groups which satisfy the same sentences with $n$ alternations of quantifiers are elementarily equivalent? We do not know the answer even in the case of metabelian groups.

(2) If two finitely generated nilpotent groups satisfy the same $\forall \exists$ sentences, are they elementarily equivalent?

In connection with these two questions, it is worth mentioning the results which have been obtained concerning the properties of polycyclic-by-finite groups which satisfy the same $\forall \exists$ sentences. By [7, Proposition 2.1, p.470], two such groups $G, H$ necessarily have the same finite images, and therefore have isomorphic profinite completions. If $G$ and $H$ are finitely generated Abelian-by-finite groups, it follows that they are elementarily equivalent, because each of them is an elementary submodel of its profinite completion according to [9, Theorem 2, p.1041].

In [15], Raphael improved [7, Proposition 2.1] by showing that, if two polycyclicby-finite groups $G, H$ satisfy the same $\forall \exists$ sentences, then, for each integer $n \geqslant 1$, there exist a subgroup $G_{n}$ of $G$ with $G_{n} \cong H$ and $\left|G: G_{n}\right|$ prime to $n$, and a subgroup $H_{n}$ of $H$ with $H_{n} \cong G$ and $\left|H: H_{n}\right|$ prime to $n$. If $G$ and $H$ are nilpotent, it follows that they have isomorphic $\pi$-localisations for each finite set $\pi$ of primes (this result was also proved in [10]). Anyhow, [8, Theorem 2.3 and Theorem 3.1, p.3] gives examples of finitely generated nilpotent groups of class 2 which have isomorphic $\pi$-localisations for each finite set $\pi$ of primes and which are not elementarily equivalent. In [16, pp.37-40], Raphael managed to show that, in one of these examples, the groups do not satisfy the same $\forall \exists$ sentences.

The definitions and results of model theory which are used here, in particular the notions of formula, sentence and elementary equivalence, are given in [2]. Concerning groups, we use the notation of [17]. In particular, we write $t(M)$ for the torsion subgroup of a finite-by nilpotent group $M$.

For each integer $n \geqslant 2$, we consider the $(n+2)$-tuples $\left(A_{1}, \ldots, A_{n+1}, f\right)$, where $A_{1}, \ldots, A_{n+1}$ are disjoint finitely generated Abelian groups and $f: A_{1} \times \cdots \times A_{n} \rightarrow A_{n+1}$ is a $n$-linear map. We also consider the triples $(A, B, f)$, where $A, B$ are disjoint finitely generated Abelian groups and $f: A^{n} \rightarrow B$ is a $n$-linear map, and the pairs $(A, f)$, where $A$ is a finitely generated Abelian group and $f: A^{n} \rightarrow A$ is a $n$-linear map.

We use the following notations, which are similar to those of [12]:

For each $(n+2)$-tuple $\left(A_{1}, \ldots, A_{n+1}, f\right)$ and for any subsets $S_{1} \subset A_{1}, \ldots, S_{n} \subset A_{n}$, we denote by $f\left(S_{1}, \ldots, S_{n}\right)$ the subgroup of $A_{n+1}$ which is generated by the elements $f\left(x_{1}, \ldots, x_{n}\right)$ for $x_{1} \in S_{1}, \ldots, x_{n} \in S_{n}$.

For any $(n+2)$-tuples $A=\left(A_{1}, \ldots, A_{n+1}, f\right)$ and $B=\left(B_{1}, \ldots, B_{n+1}, g\right)$, we consider the direct product $A \times B=\left(A_{1} \times B_{1}, \ldots, A_{n+1} \times B_{n+1}, h\right)$, with $h\left(\left(x_{1}, y_{1}\right), \ldots,\left(x_{n}, y_{n}\right)\right)=$ $\left(f\left(x_{1}, \ldots, x_{n}\right), g\left(y_{1}, \ldots, y_{n}\right)\right)$ for any elements $x_{1} \in A_{1}, y_{1} \in B_{1}, \ldots, x_{n} \in A_{n}, y_{n} \in B_{n}$. 
We define in a similar way the direct product of two triples $(A, B, f)$ and $(C, D, g)$, or the direct product of two pairs $(A, f)$ and $(B, g)$.

For each $(n+2)$-tuple $\left(A_{1}, \ldots, A_{n+1}, f\right)$ and for each $i \in\{1, \ldots, n\}$, we write $\operatorname{ker}_{i}(f)=\left\{x \in A_{i} \mid f\left(A_{1}, \ldots, A_{i-1}, x, A_{i+1}, \ldots, A_{n}\right)=0\right\}$. For a triple $(A, B, f)$ or a pair $(A, f)$, we consider $\operatorname{ker}(f)=\operatorname{ker}_{1}(f) \cap \cdots \cap \operatorname{ker}_{n}(f)$.

We interpret the $n$-linear map with the $n$-placed functional symbol $L$. For each of the $n+1$ groups, we introduce a 2-placed functional symbol for the addition, a 1-placed functional symbol for the minus operation and a constant symbol for the zero element. The universe of a $(n+2)$-tuple $\left(A_{1}, \ldots, A_{n+1}, f\right)$ is $A_{1} \cup \ldots \cup A_{n+1}$; consequently, the functions that we consider are not defined everywhere.

For reasons of convenience, we write the formulas with the symbols,,+- 0 for each of the $n+1$ groups. In order to avoid misunderstandings, the name of each variable is followed by the index $1, \ldots, n+1$ according as it concerns the elements of the first, ..., $(n+1)$-th group.

We adopt similar conventions for triples. Here, the language consists of the $n$-placed functional symbol $L$ for the $n$-linear map and, for each of the two groups, a 2-placed functional symbol, a 1-placed functional symbol and a constant symbol. The universe of a triple $(A, B, f)$ is $A \cup B$.

Concerning pairs, we use the language which consists of the $n$-placed functional symbol $L$ for the $n$-linear map and, for the group, the 2-placed functional symbol + , the 1 -placed functional symbol - and the constant symbol 0 . The universe of a pair $(A, f)$ is $A$.

In [12], we proved the following result:

THEOREM 1. For each integer $n \geqslant 2$, for each $(n+2)$-tuple $A=\left(A_{1}, \ldots, A_{n+1}, f\right)$, and for each integer $m \geqslant 1$ such that $m \mathrm{t}\left(A_{1}\right)=\ldots=m \mathrm{t}\left(A_{n+1}\right)=0$, there exist a first-order formula $\varphi_{m}\left(\bar{u}_{1}, \ldots, \bar{u}_{n+1}\right)$ and some sequences $\bar{x}_{1} \subset A_{1}, \ldots, \bar{x}_{n+1} \subset A_{n+1}$ such that:

(1) A satisfies $\varphi_{m}\left(\bar{x}_{1}, \ldots, \bar{x}_{n+1}\right)$;

(2) for each $(n+2)$-tuple $B=\left(B_{1}, \ldots, B_{n+1}, g\right)$ such that $m \mathrm{t}\left(B_{1}\right)=\ldots=$ $m \mathrm{t}\left(B_{n+1}\right)=0$, and for any sequences $\bar{y}_{1} \subset B_{1}, \ldots, \bar{y}_{n+1} \subset B_{n+1}$, if $B$ satisfies $\varphi_{m}\left(\bar{y}_{1}, \ldots, \bar{y}_{n+1}\right)$, then, for each $i \in\{1, \ldots, n\}, B_{i}$ is generated by $\bar{y}_{i}$ and $\operatorname{ker}_{i}(g)$.

In [11] and [12], we used this theorem in order to give characterisations of elementary equivalence for several classes of structures:

\section{Corollary 1.}

(1) [11] Two finitely generated finite-by-nilpotent groups $G, H$ are elementarily equivalent if and only if $\mathbf{Z} \times G$ and $\mathbf{Z} \times H$ are isomorphic. 
(2) [12] Two $(n+2)$-tuples $A=\left(A_{1}, \ldots, A_{n+1}, f\right)$ and $B=\left(B_{1}, \ldots, B_{n+1}, g\right)$ are elementarily equivalent if and only if $(\mathbf{Z}, \ldots, \mathbf{Z}, 0,0) \times A$ and $(\mathbf{Z}, \ldots, \mathbf{Z}$, $0,0) \times B$ are isomorphic.

(3) [12] Two triples $(A, B, f)$ and $(C, D, g)$ are elementarily equivalent if and only if $(\mathbf{Z}, 0,0) \times(A, B, f)$ and $(\mathrm{Z}, 0,0) \times(C, D, g)$ are isomorphic.

(4) [12] Two pairs $(A, f)$ and $(B, g)$ are elementarily equivalent if and only if $(\mathrm{Z}, 0) \times(A, f)$ and $(\mathrm{Z}, 0) \times(B, g)$ are isomorphic.

For each class, we proved that elementary equivalence does not imply isomorphism. In particular, we gave an example of two nonisomorphic finitely generated torsion-free nilpotent groups of class 3 which are elementarily equivalent, and an example of two nonisomorphic finitely generated torsion-free Lie rings which are elementarily equivalent. On the other hand, we showed that, in (2) and (3), elementary equivalence implies isomorphism if $f\left(A_{1}, \ldots, A_{n}\right)$ (respectively $\left.f\left(A^{n}\right)\right)$ is torsion-free.

In the present paper, we give a simpler proof of Theorem 1, which yields the following strengthening:

TheOREM 2. In Theorem 1, it is possible to choose a formula $\varphi_{m}$ which is a conjunction of formulas of the form

$$
(\forall \bar{v})\left(\zeta\left(\bar{u}_{1}, \ldots, \bar{u}_{n+1}, \bar{v}\right) \vee(\exists \bar{w}) \eta\left(\bar{u}_{1}, \ldots, \bar{u}_{n+1}, \bar{v}, \bar{w}\right)\right)
$$

with $\eta$ positive and $\zeta, \eta$ quantifier-free.

Then, we deduce the following result from Theorem 2 and Corollary 1:

Corollary 2. In Corollary 1 , the groups $G, H$ of (1), the $(n+2)$-tuples $A, B$ of (2), the triples $(A, B, f)$ and $(C, D, g)$ of (3), the $(A, f)$ and $(B, g)$ of (4), are elementarily equivalent if and only if they satisfy the same $\exists \forall \exists$ sentences.

The proof of Corollary 2 yields a more precise result:

\section{Corollary 3.}

(1) For each finitely generated finite-by-nilpotent group $G$ and for any integers $c, m \geqslant 1$, if $\Gamma_{c+1}(G)$ is finite and $\mathrm{t}\left(\Gamma_{i}(G) / \Gamma_{i+1}(G)\right)^{m}=1$ for $1 \leqslant i \leqslant c$ then there exists a $\exists \forall \exists$ sentence which characterises $G$ among the finitely generated finite-by-nilpotent groups $H$ such that $\mathrm{t}\left(\Gamma_{i}(H) / \Gamma_{i+1}(H)\right)^{m}=1$ for $1 \leqslant i \leqslant c$.

(2) For each $(n+2)$-tuple $A=\left(A_{1}, \ldots, A_{n+1}, f\right)$ and for each integer $m \geqslant 1$, if $m \mathrm{t}\left(A_{1}\right)=\ldots=m \mathrm{t}\left(A_{n+1}\right)=0$, then there exists a $\exists \forall \exists$ sentence which characterises $A$ among the $(n+2)$-tuples $B=\left(B_{1}, \ldots, B_{n+1}, g\right)$ such that $m \mathrm{t}\left(B_{1}\right)=\ldots=m \mathrm{t}\left(B_{n+1}\right)=0$. The same result is true for the triples $(A, B, f)$ (respectively the pairs $(A, f)$ ) if we replace the property $m \mathrm{t}\left(A_{1}\right)=\ldots=m \mathrm{t}\left(A_{n+1}\right)=0$ by $m \mathrm{t}(A)=m \mathrm{t}(B)=0$ (respectively $m \mathrm{t}(A)=0)$.

In the proof of Theorem 2, we associate a commutative ring to each $(n+2)$-tuple. In the proof of Corollaries 2 and 3, we consider, for each finitely generated finite-by-nilpotent group, some alternating bilinear maps which are defined from the map $(x, y) \rightarrow[x, y]$. In 
[11] and [12], we already used similar arguments, as well as Myasnikov in [4], [5] and [6].

PROOF OF THEOREM 2: For the sake of brevity, we write the proof with $n=2$. For each $i \in\{1,2,3\}$, we consider a sequence $\bar{x}_{i}=\left(x_{i, 1}, \ldots, x_{i, m(i)}\right)$ which generates $A_{i}$, and a sequence of variables $\bar{u}_{i}=\left(u_{i, 1}, \ldots, u_{i, m(i)}\right)$. The proof of the existence of the formulas $\varphi_{m}$ is based on the two following claims:

Claim 1. For each integer $m \geqslant 2$ such that $m \mathrm{t}\left(A_{1}\right)=m \mathrm{t}\left(A_{2}\right)=m \mathrm{t}\left(A_{3}\right)=0$, there exists a conjunction of $\sharp$ formulas $\chi_{m}\left(\bar{u}_{1}, \bar{u}_{2}, \bar{u}_{3}\right)$ such that:

(1) $A$ satisfies $\chi_{m}\left(\bar{x}_{1}, \bar{x}_{2}, \bar{x}_{3}\right)$;

(2) for each quadruple $B=\left(B_{1}, B_{2}, B_{3}, g\right)$ such that $m \mathrm{t}\left(B_{1}\right)=m \mathrm{t}\left(B_{2}\right)=$ $m \mathrm{t}\left(B_{3}\right)=0$, and for any sequences $\bar{y}_{1} \subset B_{1}, \bar{y}_{2} \subset B_{2}, \bar{y}_{3} \subset B_{3}$, if $B$ satisfies $\chi_{m}\left(\bar{y}_{1}, \bar{y}_{2}, \bar{y}_{3}\right)$, then there exists an injective homomorphism $\theta=$ $\left(\theta_{1}, \theta_{2}, \theta_{3}\right): A \rightarrow B$ such that, for each $i \in\{1,2,3\}, \theta_{i}\left(\bar{x}_{i}\right)=\bar{y}_{i}$ and $\left|B_{i} / \theta_{i}\left(A_{i}\right)\right|$ is prime to $m$.

Claim 2. There exist an integer $m_{0} \geqslant 2$ and a conjunction of $\sharp$ formulas $\psi\left(\bar{u}_{1}, \bar{u}_{2}\right)$ such that:

(1) $A$ satisfies $\psi\left(\bar{x}_{1}, \vec{x}_{2}\right)$;

(2) for each quadruple $B=\left(B_{1}, B_{2}, B_{3}, g\right)$ with $A \subset B$ and $B_{1} / A_{1}, B_{2} / A_{2}$ finite, if $B$ satisfies $\psi\left(\bar{x}_{1}, \bar{x}_{2}\right)$, then, for each $i \in\{1,2\},\left|B_{i} /\left\langle A_{i}, \operatorname{ker}_{i}(g)\right\rangle\right|$ divides $m_{0}$.

First, we show that, if the two claims are true, then $\varphi_{m}$ exists for each integer $m \geqslant 2$ such that $m \mathrm{t}\left(A_{1}\right)=m \mathrm{t}\left(A_{2}\right)=m \mathrm{t}\left(A_{3}\right)=0$ and $m_{0}$ divides $m$ (we take $\varphi_{m}=\varphi_{m_{0} m}$ if $m_{0}$ does not divide $m$ ).

We consider the formula $\varphi_{m}\left(\bar{u}_{1}, \bar{u}_{2}, \bar{u}_{3}\right)=\chi_{m}\left(\bar{u}_{1}, \bar{u}_{2}, \bar{u}_{3}\right) \wedge \psi\left(\bar{u}_{1}, \bar{u}_{2}\right)$, which is satisfied by $\bar{x}_{1}, \bar{x}_{2}, \bar{x}_{3}$ in A. For each quadruple $B=\left(B_{1}, B_{2}, B_{3}, g\right)$ such that $m \mathrm{t}\left(B_{1}\right)=m \mathrm{t}\left(B_{2}\right)=$ $m \mathrm{t}\left(B_{3}\right)=0$, and for any sequences $\bar{y}_{1} \subset B_{1}, \bar{y}_{2} \subset B_{2}, \bar{y}_{3} \subset B_{3}$, if $B$ satisfies $\varphi_{m}\left(\bar{y}_{1}, \bar{y}_{2}, \bar{y}_{3}\right)$, then there exists an injective homomorphism $\theta=\left(\theta_{1}, \theta_{2}, \theta_{3}\right): A \rightarrow B$ such that, for each $i \in\{1,2\}, \theta_{i}\left(\bar{x}_{i}\right)=\bar{y}_{i}$ and $\left|B_{i} / \theta_{i}\left(A_{i}\right)\right|$ is prime to $m$. For each $i \in\{1,2\}$, we have $B_{i}=\left\langle\theta_{i}\left(A_{i}\right), \operatorname{ker}_{i}(g)\right\rangle=\left\langle\bar{y}_{i}, \operatorname{ker}_{i}(g)\right\rangle$ since $\left|B_{i} /\left\langle\theta_{i}\left(A_{i}\right), \operatorname{ker}_{i}(g)\right\rangle\right|$ is prime to $m$ and divides $m$.

Then, we prove Claim 1. For each $i \in\{1,2,3\}$, there exist two integers $q(i) \leqslant r(i)$ and some terms $\rho_{i, 1}\left(\bar{u}_{i}\right), \ldots, \rho_{i, r(i)}\left(\bar{u}_{i}\right)$ such that $\mathrm{t}\left(A_{i}\right)=\left\{\rho_{i, 1}\left(\bar{x}_{i}\right), \ldots, \rho_{i, q(i)}\left(\bar{x}_{i}\right)\right\}$ and such that $A_{i}$ is the disjoint union of the subsets $\rho_{i, j}\left(\bar{x}_{i}\right)+m A_{i}$ for $1 \leqslant j \leqslant r(i)$. For each $i \in\{1,2,3\}$, there are also some terms $\sigma_{i, 1}\left(\bar{u}_{i}\right), \ldots, \sigma_{i, s(i)}\left(\bar{u}_{i}\right)$ such that $\left\langle\bar{x}_{i} ; \sigma_{i, 1}\left(\bar{x}_{i}\right), \ldots, \sigma_{i, s(i)}\left(\bar{x}_{i}\right)\right\rangle$ is a presentation of $A_{i}$. For each $i \in\{1, \ldots, m(1)\}$ and each $j \in\{1, \ldots, m(2)\}$, there exists a term $\tau_{i, j}\left(\bar{u}_{3}\right)$ such that $f\left(x_{1, i}, x_{2, j}\right)=\tau_{i, j}\left(\bar{x}_{3}\right)$. 
The conjunction of $\sharp$ formulas $\chi_{m}\left(\bar{u}_{1}, \bar{u}_{2}, \bar{u}_{3}\right)$ below is satisfied by $\bar{x}_{1}, \bar{x}_{2}, \bar{x}_{3}$ :

$$
\begin{aligned}
{\left[\mathbb{M}_{1 \leqslant i \leqslant 3}\left(\forall v_{i}\right)\right.} & {\left.\left[m v_{i}=0 \leftrightarrow\left(\mathbb{W}_{1 \leqslant j \leqslant q(i)} v_{i}=\rho_{i, j}\left(\bar{u}_{i}\right)\right)\right]\right] } \\
& \wedge\left[\mathbb{M}_{1 \leqslant i \leqslant 3}\left(\forall v_{i}\right)\left(\exists w_{i}\right)\left(W_{1 \leqslant j \leqslant r(i)} v_{i}=\rho_{i, j}\left(\bar{u}_{i}\right)+m w_{i}\right)\right] \\
& \wedge\left[\mathbb{M}_{1 \leqslant i \leqslant 3}^{1 \leqslant j \leqslant r(i)}\left(\forall v_{i}\right) \neg\left(\rho_{i, j}\left(\bar{u}_{i}\right)=\rho_{i, k}\left(\bar{u}_{i}\right)+m v_{i}\right)\right] \\
& \wedge\left[\mathbb{M}_{1 \leqslant i \leqslant 3}^{1 \leqslant j \leqslant s(i)} \sigma_{i, j}\left(\bar{u}_{i}\right)=0\right] \wedge\left[\mathbb{M}_{1 \leqslant i \leqslant m(1)}^{1 \leqslant j(1)} L\left(u_{1, i}, u_{2, j}\right)=\tau_{i, j}\left(\bar{u}_{3}\right)\right] .
\end{aligned}
$$

For each quadruple $B=\left(B_{1}, B_{2}, B_{3}, g\right)$ and for any sequences $\bar{y}_{1} \subset B_{1}, \bar{y}_{2} \subset B_{2}, \bar{y}_{3} \subset B_{3}$ which satisfy $\chi_{m}$ in $B$, there exists a unique homomorphism $\theta=\left(\theta_{1}, \theta_{2}, \theta_{3}\right): A \rightarrow B$ such that $\theta_{i}\left(\bar{x}_{i}\right)=\bar{y}_{i}$ for each $i \in\{1,2,3\}$. For each $i \in\{1,2,3\}, \theta_{i}$ induces an isomorphism from $A_{i} / m A_{i}$ to $B_{i} / m B_{i}$. If $m \mathrm{t}\left(B_{i}\right)=0$, then $\theta_{i}$ also induces an isomorphism from $\mathrm{t}\left(A_{i}\right)$ to $\mathrm{t}\left(B_{i}\right)$. It follows that $\theta_{i}$ is injective and $\left|B_{i} / \theta_{i}\left(A_{i}\right)\right|$ is prime to $m$ (the details of the argument are given in $[10$, p.66]).

Now, we observe that $f\left(A_{1}, A_{2}\right)=\left\{\sum_{i=1}^{m(1)} f\left(x_{1, i}, y_{2, i}\right) \mid y_{2,1}, \ldots, y_{2, m(1)} \in A_{2}\right\}$, and we show that it suffices to prove Claim 2 in a weaker form:

Claim 3. If $f$ is nondegenerate and $f\left(A_{1}, A_{2}\right)=A_{3}$, then there exist an integer $m$ and a conjunction of $\sharp$ formulas $\psi\left(\bar{u}_{1}, \bar{u}_{2}\right)$ such that:

(1) A satisfies $\psi\left(\bar{x}_{1}, \bar{x}_{2}\right)$;

(2) for each quadruple $B=\left(B_{1}, B_{2}, B_{3}, g\right)$ which satisfies the list of conditions

(*) below:

$A \subset B$ and $B_{1} / A_{1}, B_{2} / A_{2}$ finite,

$$
\begin{aligned}
& B_{3}=g\left(B_{1}, B_{2}\right)=\left\{\sum_{i=1}^{m(1)} g\left(x_{1, i}, y_{2, i}\right) \mid y_{2,1}, \ldots, y_{2, m(1)} \in B_{2}\right\}, \\
& x_{1}=0 \text { for each } x_{1} \in B_{1} \text { such that } g\left(x_{1}, \bar{x}_{2}\right)=0 \\
& x_{2}=0 \text { for each } x_{2} \in B_{2} \text { such that } g\left(\bar{x}_{1}, x_{2}\right)=0,
\end{aligned}
$$

if $B$ satisfies $\psi\left(\bar{x}_{1}, \bar{x}_{2}\right)$, then, for each $i \in\{1,2\},\left|B_{i} / A_{i}\right|$ divides $m$.

In order to prove that Claim 3 implies Claim 2, for each quadruple $B=\left(B_{1}, B_{2}, B_{3}, g\right)$, we consider the quadruple $B^{*}=\left(B_{1}^{*}, B_{2}^{*}, B_{3}^{*}, g^{*}\right)$, where $B_{1}^{*}=B_{1} / \operatorname{ker}_{1}(g), B_{2}^{*}=$ $B_{2} / \operatorname{ker}_{2}(g), B_{3}^{*}=g\left(B_{1}, B_{2}\right)$, and $g^{*}$ is the bilinear map from $B_{1}^{*} \times B_{2}^{*}$ to $B_{3}^{*}$ which is induced by $g$. For each $x \in B_{1} \cup B_{2}$, we denote by $x^{*}$ the image of $x$ in $B^{*}$.

According to Claim 3 , there exist an integer $m$ and a conjunction of $\sharp$ formulas $\psi^{*}\left(\bar{u}_{1}, \bar{u}_{2}\right)$ such that:

(1) $A^{*}$ satisfies $\psi^{*}\left(\bar{x}_{1}^{*}, \bar{x}_{2}^{*}\right)$;

(2) for each quadruple $C=\left(C_{1}, C_{2}, C_{3}, h\right)$ which satisfies (*) relative to $A^{*}$, if $C$ satisfies $\psi^{*}\left(\bar{x}_{1}^{*}, \bar{x}_{2}^{*}\right)$, then, for each $i \in\{1,2\},\left|C_{i} / A_{i}^{*}\right|$ divides $m$. 
We consider the conjunction of $\sharp$ formulas $\psi\left(\bar{u}_{1}, \bar{u}_{2}\right)=\psi_{0}\left(\bar{u}_{1}, \bar{u}_{2}\right) \wedge \psi_{1}\left(\bar{u}_{1}, \bar{u}_{2}\right)$, where $\psi_{0}\left(\bar{u}_{1}, \bar{u}_{2}\right)$ is the formula

$$
\begin{aligned}
\left(\forall v_{1}\right)\left(L\left(v_{1}, \bar{u}_{2}\right)\right. & \left.=0 \rightarrow\left(\forall v_{2}\right) L\left(v_{1}, v_{2}\right)=0\right) \\
& \wedge\left(\forall v_{2}\right)\left(L\left(\bar{u}_{1}, v_{2}\right)=0 \rightarrow\left(\forall v_{1}\right) L\left(v_{1}, v_{2}\right)=0\right) \\
& \wedge\left(\forall v_{1} \forall v_{2}\right)\left(\exists v_{2,1} \ldots \exists v_{2, m(1)}\right) L\left(v_{1}, v_{2}\right)=\sum_{i=1}^{m(1)} L\left(u_{1, i}, v_{2, i}\right),
\end{aligned}
$$

and $\psi_{1}\left(\bar{u}_{1}, \bar{u}_{2}\right)$ is the conjunction of $\sharp$ formulas which is obtained from $\psi^{*}\left(\bar{u}_{1}, \bar{u}_{2}\right)$ by replacing successively:

(a) each atomic subformula $\mathrm{t}\left(\bar{v}_{1}\right)=0$ (respectively $\left.\mathrm{t}\left(\bar{v}_{2}\right)=0\right)$ by $L\left(\mathrm{t}\left(\bar{v}_{1}\right), \bar{u}_{2}\right)=$ 0 (respectively $L\left(\bar{u}_{1}, \mathrm{t}\left(\bar{v}_{2}\right)\right)=0$ );

(b) each subformula $\left(\exists v_{3}\right) \theta$ (respectively $\left.\left(\forall v_{3}\right) \theta\right)$ by $\left(\exists v_{3}\right)\left(\exists v_{2,1} \ldots \exists v_{2, m(1)}\right)\left[v_{3}=\right.$ $\left.\sum_{i=1}^{m(1)} L\left(u_{1, i}, v_{2, i}\right) \wedge \theta\right]$ (respectively $\left(\forall v_{3}\right)\left(\forall v_{2,1} \ldots \forall v_{2, m(1)}\right)\left[v_{3}=\sum_{i=1}^{m(1)} L\left(u_{1, i}, v_{2, i}\right)\right.$ $\rightarrow \theta]$ ).

The quadruple $A$ satisfies $\psi\left(\bar{x}_{1}, \bar{x}_{2}\right)$. For each quadruple $B=\left(B_{1}, B_{2}, B_{3}, g\right)$ with $A \subset B$ and $B_{1} / A_{1}, B_{2} / A_{2}$ finite, if $B$ satisfies $\psi\left(\bar{x}_{1}, \bar{x}_{2}\right)$, then $B^{*}$ satisfies $\psi^{*}\left(\bar{x}_{1}^{*}, \bar{x}_{2}^{*}\right)$. It follows that, for each $i \in\{1,2\},\left|B_{i}^{*} / A_{i}^{*}\right|=\left|B_{i} /\left\langle A_{i}, \operatorname{ker}_{i}(g)\right\rangle\right|$ divides $m$.

Finally, we prove Claim 3. For each quadruple $B=\left(B_{1}, B_{2}, B_{3}, g\right)$ which satisfies (*), we consider the set $R_{B}$ which consists of the triples $\left(\theta_{1}, \theta_{2}, \theta_{3}\right) \in \operatorname{End}\left(B_{1}\right) \times \operatorname{End}\left(B_{2}\right) \times$ End $\left(B_{3}\right)$ with $g\left(\theta_{1}\left(y_{1}\right), y_{2}\right)=g\left(y_{1}, \theta_{2}\left(y_{2}\right)\right)=\theta_{3}\left(y_{3}\right)$ for any elements $y_{1} \in B_{1}, y_{2} \in B_{2}$ and $y_{3} \in B_{3}$ such that $g\left(y_{1}, y_{2}\right)=y_{3}$. We define a commutative ring structure on $R_{B}$ by writing $\theta+\theta^{\prime}=\left(\theta_{1}+\theta_{1}^{\prime}, \theta_{2}+\theta_{2}^{\prime}, \theta_{3}+\theta_{3}^{\prime}\right)$ and $\theta \circ \theta^{\prime}=\left(\theta_{1} \circ \theta_{1}^{\prime}, \theta_{2} \circ \theta_{2}^{\prime}, \theta_{3} \circ \theta_{3}^{\prime}\right)$ for $\theta=\left(\theta_{1}, \theta_{2}, \theta_{3}\right)$ and $\theta^{\prime}=\left(\theta_{1}^{\prime}, \theta_{2}^{\prime}, \theta_{3}^{\prime}\right)$. This follows since any triples $\theta=\left(\theta_{1}, \theta_{2}, \theta_{3}\right)$ and $\theta^{\prime}=\left(\theta_{1}^{\prime}, \theta_{2}^{\prime}, \theta_{3}^{\prime}\right)$ in $R_{B}$ necessarily satisfy

$$
\begin{aligned}
g\left(\theta_{1}^{\prime}\left(\theta_{1}\left(x_{1}\right)\right), x_{2}\right) & =\theta_{3}^{\prime}\left(g\left(\theta_{1}\left(x_{1}\right), x_{2}\right)\right)=\theta_{3}^{\prime}\left(\theta_{3}\left(g\left(x_{1}, x_{2}\right)\right)\right) \\
& =\theta_{3}^{\prime}\left(g\left(x_{1}, \theta_{2}\left(x_{2}\right)\right)\right)=g\left(x_{1}, \theta_{2}^{\prime}\left(\theta_{2}\left(x_{2}\right)\right)\right) \text { and } \\
g\left(\theta_{1}^{\prime}\left(\theta_{1}\left(x_{1}\right)\right), x_{2}\right) & =g\left(x_{1}, \theta_{2}^{\prime}\left(\theta_{2}\left(x_{2}\right)\right)\right)=g\left(\theta_{1}^{\prime}\left(x_{1}\right), \theta_{2}\left(x_{2}\right)\right) \\
& =g\left(\theta_{1}\left(\theta_{1}^{\prime}\left(x_{1}\right)\right), x_{2}\right)
\end{aligned}
$$

for $x_{1} \in B_{1}$ and $x_{2} \in B_{2}$, and therefore $\theta^{\prime} \circ \theta=\theta \circ \theta^{\prime} \in R_{B}$.

Any triple $\left(\theta_{1}, \theta_{2}, \theta_{3}\right) \in R_{B}$ is completely determined by $\theta_{1}\left(\bar{x}_{1}\right)$ or $\theta_{2}\left(\bar{x}_{2}\right)$. This follows because if, for instance, $\theta_{2}\left(\bar{x}_{2}\right)=0$, then any element $y_{1} \in B_{1}$ satisfies $g\left(\theta_{1}\left(y_{1}\right), \bar{x}_{2}\right)=$ $g\left(y_{1}, \theta_{2}\left(\bar{x}_{2}\right)\right)=g\left(y_{1}, 0\right)=0$, and therefore $\theta_{1}\left(y_{1}\right)=0$.

The group $\left(R_{B},+\right)$ is finitely generated, and the ring $R_{B}$ is Noetherian, since $B_{1}$ and $B_{2}$ are finitely generated.

We write $\bar{u}=\left(\bar{u}_{1}, \bar{u}_{2}\right)$ and $\bar{x}=\left(\bar{x}_{1}, \bar{x}_{2}\right)$. We identify each $\left(\theta_{1}, \theta_{2}, \theta_{3}\right) \in R_{B}$ with $\left(\theta_{1}\left(\bar{x}_{1}\right), \theta_{2}\left(\bar{x}_{2}\right)\right)$. In particular, we identify $\left(\operatorname{Id}_{B_{1}}, \operatorname{Id}_{B_{2}}, \operatorname{Id}_{B_{3}}\right)$ and $\bar{x}$. For each pair $\bar{y}=$ 
$\left(\bar{y}_{1}, \bar{y}_{2}\right)$ with $\bar{y}_{1}=\left(y_{1,1}, \ldots, y_{1, m(1)}\right) \subset B_{1}$ and $\bar{y}_{2}=\left(y_{2,1}, \ldots, y_{2, m(2)}\right) \subset B_{2}$, we write $\bar{y} \in R_{B}$ if there exists a triple $\left(\theta_{1}, \theta_{2}, \theta_{3}\right) \in R_{B}$ such that $\theta_{1}\left(\bar{x}_{1}\right)=\bar{y}_{1}$ and $\theta_{2}\left(\bar{x}_{2}\right)=\bar{y}_{2}$.

For each sequence of variables $\bar{v}=\left(\bar{v}_{1}, \bar{v}_{2}\right)$ with $\bar{v}_{1}=\left(v_{1,1}, \ldots, v_{1, m(1)}\right)$ and $\bar{v}_{2}=$ $\left(v_{2,1}, \ldots, v_{2, m(2)}\right)$, and for any variables $u_{1}, u_{2}, v_{1}, v_{2}$, we denote by $\bar{v} u_{1}=v_{1}$ and $\bar{v} u_{2}=v_{2}$ the positive quantifier-free formulas

$$
\mathbb{M}_{1 \leqslant j \leqslant m(2)} L\left(u_{1}, v_{2, j}\right)=L\left(v_{1}, u_{2, j}\right) \text { and } \mathbb{M}_{1 \leqslant i \leqslant m(1)} L\left(v_{1, i}, u_{2}\right)=L\left(u_{1, i}, v_{2}\right) .
$$

For each $i \in 1,2$, we consider some terms $\rho_{i, 1}\left(\bar{u}_{i}\right), \ldots, \rho_{i, p(i)}\left(\bar{u}_{i}\right)$ which define a presentation of $A_{i}$ on $\bar{x}_{i}$. We also consider some terms

$$
\rho_{3,1}\left(L\left(u_{1, i}, u_{2, j}\right)_{1 \leqslant i \leqslant m(1)}^{1 \leqslant j \leqslant m(2)}\right), \ldots, \rho_{3, p(3)}\left(L\left(u_{1, i}, u_{2, j}\right)_{1 \leqslant i \leqslant m(1)}^{1 \leqslant j \leqslant m(2)}\right)
$$

which define a presentation of $A_{3}$ on $f\left(x_{1, i}, x_{2, j}\right)_{1 \leqslant i \leqslant m(1)}^{1 \leqslant j \leqslant m(2)}$. For each pair $\bar{y}=\left(\bar{y}_{1}, \bar{y}_{2}\right)$ with $\bar{y}_{1}=\left(y_{1,1}, \ldots, y_{1, m(1)}\right) \subset A_{1}$ and $\bar{y}_{2}=\left(y_{2,1}, \ldots, y_{2, m(2)}\right) \subset A_{2}$, we have $\bar{y} \in R_{A}$ if and only if $(\bar{x}, \bar{y})$ satisfies the positive quantifier-free formula $\lambda(\bar{u}, \bar{v})$ below:

$$
\begin{aligned}
\left(\mathbb{N}_{1 \leqslant i \leqslant m(1)}^{1 \leqslant j \leqslant m(2)} L\left(u_{1, i}, v_{2, j}\right)=L\left(v_{1, i}, u_{2, j}\right)\right) & \wedge\left(\mathbb{M}_{1 \leqslant i \leqslant p(1)} \rho_{1, i}\left(\bar{v}_{1}\right)=0\right) \\
& \wedge\left(\mathbb{M}_{1 \leqslant j \leqslant p(2)} \rho_{2, j}\left(\bar{v}_{2}\right)=0\right) \wedge\left(\mathbb{M}_{1 \leqslant k \leqslant p(3)} \rho_{3, k}\left(L\left(v_{1, i}, u_{2, j}\right)_{1 \leqslant i \leqslant m(1)}^{1 \leqslant j \leqslant m(2)}\right)=0\right) .
\end{aligned}
$$

We denote by $\mu(\bar{u}, \bar{v})$ the formula

$$
\begin{gathered}
{\left[\mathbb{\aleph}_{i=1,2}\left(\forall u_{i}\right)\left(\exists v_{i}\right)\left(\bar{v} u_{i}=v_{i}\right)\right] \wedge\left(\forall u_{1}\right)\left(\forall v_{1}\right)\left(\forall u_{2}\right)\left(\forall v_{2}\right)\left(\forall w_{2,1} \ldots \forall w_{2, m(1)}\right)} \\
{\left[\left[L\left(u_{1}, u_{2}\right)=\sum_{i=1}^{m(1)} L\left(u_{1, i}, w_{2, i}\right) \wedge \bar{v} u_{1}=v_{1} \wedge \bar{v} u_{2}=v_{2}\right]\right.} \\
\left.\rightarrow L\left(u_{1}, v_{2}\right)=L\left(v_{1}, u_{2}\right)=\sum_{i=1}^{m(1)} L\left(v_{1, i}, w_{2, i}\right)\right]
\end{gathered}
$$

Now, let us consider a quadruple $B=\left(B_{1}, B_{2}, B_{3}, g\right)$ which satisfies (*). For each pair $\bar{y}=\left(\bar{y}_{1}, \bar{y}_{2}\right)$ with $\bar{y}_{1}=\left(y_{1,1}, \ldots, y_{1, m(1)}\right) \subset B_{1}$ and $\bar{y}_{2}=\left(y_{2,1}, \ldots, y_{2, m(2)}\right) \subset B_{2}$, if $\bar{y}$ belongs to $R_{B}$, then $(\bar{x}, \bar{y})$ satisfies $\mu(\bar{u}, \bar{v})$. Conversely, if $(\bar{x}, \bar{y})$ satisfies $\mu(\bar{u}, \bar{v})$, then we define a triple $\theta=\left(\theta_{1}, \theta_{2}, \theta_{3}\right) \in R_{B}$ with $\theta(\bar{x})=\bar{y}$ by writing $\theta_{1}\left(z_{1}\right)=\bar{y} z_{1}$, $\theta_{2}\left(z_{2}\right)=\bar{y} z_{2}$ and $\theta_{3}\left(\sum_{i=1}^{m(1)} g\left(x_{1, i}, z_{2, i}\right)\right)=\sum_{i=1}^{m(1)} g\left(y_{1, i}, z_{2, i}\right)$ for any elements $z_{1} \in B_{1}$ and $z_{2}, z_{2,1}, \ldots, z_{2, m(1)} \in B_{2}$. This follows since any element of $g\left(B_{1}, B_{2}\right)$ can be written as $\sum_{i=1}^{m(1)} g\left(x_{1, i}, z_{2, i}\right)$, and $\sum_{i=1}^{m(1)} g\left(x_{1, i}, z_{2, i}\right)=0$ implies $\sum_{i=1}^{m(1)} g\left(y_{1, i}, z_{2, i}\right)=0$.

The formula $\alpha(\bar{u})=(\forall \bar{v})(\lambda(\bar{u}, \bar{v}) \rightarrow \mu(\bar{u}, \bar{v}))$ is equivalent to a conjunction of $\sharp$ formulas. It is satisfied by $\bar{x}$ in $A$. If $\bar{x}$ satisfies $\alpha$ in $B$, then, for each pair $\bar{y}=\left(\bar{y}_{1}, \bar{y}_{2}\right)$ with $\bar{y}_{1}=\left(y_{1,1}, \ldots, y_{1, m(1)}\right) \subset B_{1}$ and $\bar{y}_{2}=\left(y_{2,1}, \ldots, y_{2, m(2)}\right) \subset B_{2}$, we have $\bar{y} \in R_{B}$ if 
and only if $(\bar{x}, \bar{y})$ satisfies $\lambda$ in $B$. In particular, we have $R_{A} \subset R_{B}$. Moreover, $R_{B} / R_{A}$ is finite since $B_{1} / A_{1}$ and $B_{2} / A_{2}$ are finite.

For each sequence $\left(\vec{y}^{i, j}\right)_{1 \leqslant i \leqslant q}^{1 \leqslant j \leqslant r(i)} \subset R_{B}$, we have $\sum_{i=1}^{q}\left(\bar{y}^{i, 1}\right) \ldots\left(\bar{y}^{i, r(i)}\right)=0$ if and only if $\left(\bar{x},\left(\bar{y}^{i, j}\right)_{1 \leqslant i \leqslant q}^{1 \leqslant j \leqslant r(i)}\right)$ satisfies, in $B$, the positive existential formula below, which we shall denote by $\sum_{i=1}^{q} \vec{v}^{i, 1} \cdots \bar{v}^{i, r(i)}=0$ :

$$
\begin{aligned}
\bigwedge_{k=1}^{m(1)}\left(\exists\left(w_{1, k}^{i, j}\right)_{1 \leqslant i \leqslant q}^{0 \leqslant j \leqslant r(i)}\right)\left[\left(\bigwedge_{i=1}^{q} w_{1, k}^{i, 0}=u_{1, k}\right)\right. & \wedge\left(\bigwedge_{1 \leqslant i \leqslant q}^{1 \leqslant j \leqslant r(i)}-^{i, j} w_{1, k}^{i, j-1}=w_{1, k}^{i, j}\right) \\
& \left.\wedge\left(\sum_{i=1}^{q} w_{1, k}^{i, r(i)}=0\right)\right] .
\end{aligned}
$$

For each $i \in\{1,2\}$, we have $A_{i}=\mathbf{Z} x_{i, 1}+\cdots+\mathbf{Z} x_{i, m(i)}$, and therefore $A_{i}=R_{A} x_{i, 1}+$ $\cdots+R_{A} x_{i, m(i)}$. So, the $\sharp$ formula $\beta(\bar{u})$ below is satisfied by $\bar{x}$ in $A$ :

$$
\mathbb{M}_{i=1,2}\left(\forall v_{i}\right)\left(\exists \bar{v}^{i, 1} \ldots \exists \bar{v}^{i, m(i)}\right)\left[\left(\mathbb{M}_{1 \leqslant j \leqslant m(i)} \lambda\left(\bar{u}, \bar{v}^{i, j}\right)\right) \wedge\left(v_{i}=\sum_{1 \leqslant j \leqslant m(i)} \bar{v}^{i, j} u_{i, j}\right)\right] .
$$

If $\bar{x}$ satisfies $\alpha \wedge \beta$ in $B$, then, for each $i \in\{1,2\}$, we have $B_{i}=R_{B} x_{i, 1}+\cdots+R_{B} x_{i, m(i)}$.

Now, we consider an integer $t \geqslant 1$ such that $\left(R_{A},+\right)$ is generated by $t$ elements, and some prime ideals $P_{1}, \ldots, P_{s}$ of $R_{A}$ such that $P_{1} \cdots P_{s}=0$. For each $i \in\{1, \ldots, s\}$, there exist some elements $\bar{x}^{i, 1}, \ldots, \bar{x}^{i, t} \in P_{i}$ such that $P_{i}=\mathbf{Z} \bar{x}^{i, 1}+\cdots+\mathbf{Z} \bar{x}^{i, t}$. For each $i \in\{1, \ldots, s\}$ and each $j \in\{1, \ldots, t\}$, there exists a sequence of terms $\bar{\tau}^{i, j}(\bar{u})=$ $\left(\tau_{k, l}^{i, j}(\bar{u})\right)_{k=1,2}^{1 \leqslant l \leqslant m(k)}$ such that $\bar{x}^{i, j}=\left(\tau_{k, l}^{i, j}(\bar{x})\right)_{k=1,2}^{1 \leqslant l \leqslant m(k)}$.

For each $i \in\{1, \ldots, s\}$, as $P_{i}=R_{A} \bar{x}^{i, 1}+\cdots+R_{A} \bar{x}^{i, t}$ is prime, the $\sharp$ formula $\pi_{i}(\bar{u})$ below is satisfied by $\bar{x}$ in $A$ :

$$
\begin{aligned}
\left(\forall \bar{v}^{1}\right)\left(\forall \bar{v}^{2}\right)\left\{\left\{\lambda\left(\bar{u}, \bar{v}^{1}\right) \wedge \lambda\left(\bar{u}, \bar{v}^{2}\right)\right.\right. & \wedge\left(\exists \bar{w}^{1} \ldots \exists \bar{w}^{t}\right)\left[\left(M_{1 \leqslant k \leqslant t} \lambda\left(\bar{u}, \bar{w}^{k}\right)\right)\right. \\
& \left.\left.\wedge\left(\bar{v}^{1} \bar{v}^{2}=\sum_{1 \leqslant k \leqslant t} \bar{w}^{k} \bar{\tau}^{i, k}(\bar{u})\right)\right]\right\} \\
\rightarrow\left(\exists \bar{w}^{1} \ldots \exists \bar{w}^{t}\right)\left[\left(\mathbb{M}_{1 \leqslant k \leqslant t} \lambda\left(\bar{u}, \bar{w}^{k}\right)\right)\right. & \left.\left.\wedge\left(\mathbb{W}_{j=1,2} \bar{v}^{j}=\sum_{1 \leqslant k \leqslant t} \bar{w}^{k} \bar{\tau}^{i, k}(\bar{u})\right)\right]\right\} .
\end{aligned}
$$

We denote by $\gamma(\bar{u})$ the conjunction of the formulas $\pi_{i}(\bar{u})$. If $\bar{x}$ satisfies $\alpha \wedge \beta \wedge \gamma$ in $B$, then, for each $i \in\{1, \ldots, s\}$, the ideal $Q_{i}=R_{B} \bar{x}^{i, 1}+\cdots+R_{B} \bar{x}^{i, t}$ is prime, we have $P_{i} \subset Q_{i}$, and $Q_{i} / P_{i}$ is finite since $R_{B} / R_{A}$ is finite. Moreover, the equalities $\bar{x}^{1, i(1)} \ldots \bar{x}^{s, i(s)}=0$ for $1 \leqslant i(1), \ldots, i(s) \leqslant t$ imply $Q_{1} \cdots Q_{s}=0$.

For each $i \in\{1, \ldots, s\}$ such that $R_{A} / P_{i}$ is finite, there exist some sequences of terms $\bar{\sigma}^{i, 1}(\bar{u}), \ldots, \bar{\sigma}^{i, q(i)}(\bar{u})$ such that $R_{A}$ is the union of the subsets $\bar{\sigma}^{i, j}(\bar{x})+P_{i}$. The $\sharp$ formula $\delta_{i}(\bar{u})$ below is satisfied by $\bar{x}$ in $A$ :

$$
(\forall \bar{v})\left[W_{1 \leqslant j \leqslant q(i)}\left(\exists \bar{w}^{1} \ldots \exists \bar{w}^{l}\right)\left(\bar{v}=\bar{\sigma}^{i, j}(\bar{u})+\sum_{1 \leqslant h \leqslant t} \bar{w}^{h} \bar{\tau}^{i, h}(\bar{u})\right)\right] .
$$


If $\bar{x}$ satisfies $\alpha \wedge \beta \wedge \gamma \wedge \delta_{i}$ in $B$, then we have $R_{B}=R_{A}+Q_{i}$.

We denote by $\delta$ the conjunction of the formulas $\delta_{i}$ for $R_{A} / P_{i}$ finite, and we write $\psi=\alpha \wedge \beta \wedge \gamma \wedge \delta$. This formula is satisfied by $\bar{x}$ in $\mathrm{A}$. We consider the quadruples $B=\left(B_{1}, B_{2}, B_{3}, g\right)$ which satisfy $(*)$ and such that $\bar{x}$ satisfies $\psi$ in $\mathrm{B}$. It suffices to show that, concerning such quadruples, there exists a bound for $\left|B_{1} / A_{1}\right|$ and $\left|B_{2} / A_{2}\right|$ which only depends on $\mathrm{A}$.

For each $i \in\{1,2\}$, we have $A_{i}=R_{A} x_{i, 1}+\cdots+R_{A} x_{i, m(i)}$ and $B_{i}=R_{B} x_{i, 1}+\cdots+$ $R_{B} x_{i, m(i)}$; it follows $\left|B_{i} / A_{i}\right| \leqslant\left|R_{B} / R_{A}\right|^{m(i)}$. Consequently, it suffices to give a bound for $\left|R_{B} / R_{A}\right|$ which only depends on $A$. This bound is given by the following:

LEMMA . Let $R$ be a commutative ring with a finitely generated additive group. Let $P_{1}, \ldots, P_{k}$ be prime ideals of $R$ with $P_{1} \ldots P_{k}=0$. Then, there exists an integer $m$ such that, for every commutative ring $S$ with $R \subset S$ and $|S / R|$ finite, we have $|S / R| \leqslant m$ if the following conditions are satisfied for $1 \leqslant i \leqslant k$ :

(1) $S P_{i}$ is a prime ideal of $S$;

(2) $R / P_{i}$ finite implies $S=R+S P_{i}$.

Proof: For each $i \in\{1, \ldots, k\}$, we write $Q_{i}=S P_{i}, R_{i}=R / P_{i}$ and $S_{i}=S / Q_{i}$. We first show that there is a bound for $\left|S /\left(R+Q_{i}\right)\right|$ which only depends on $R$. If $R_{i}$ is finite, we have $S=R+Q_{i}$ by (2). So, we can suppose $R_{i}$ infinite. Then, $S_{i}$ is also infinite since $Q_{i} / P_{i}$ is finite like $S / R$. It follows that the finitely generated Abelian groups $\left(R_{i},+\right)$ and $\left(S_{i},+\right)$ are torsion-free, since $R_{i}$ and $S_{i}$ are commutative integral domains. By [3, Proposition 3, p.163 and Proposition 5, p.165] $K_{i}=\left\{a / n \mid a \in R_{i}\right.$ and $\left.n \in \mathbf{N}^{*}\right\}$ and $L_{i}=\left\{a / n \mid a \in S_{i}\right.$ and $\left.n \in \mathbf{N}^{*}\right\}$ are algebraic extensions of finite degree of $\mathbf{Q}$, and $R_{i}$ (respectively $S_{i}$ ) is a subgroup of finite index in the integer ring $\bar{R}_{i}$ of $K_{i}$ (respectively $\bar{S}_{i}$ of $\left.L_{i}\right)$.

We have $Q_{i} \cap R=P_{i}$ since $Q_{i} / P_{i}$ is finite and $R / P_{i}$ is torsion-free. So, the inclusion $R \subset S$ induces an injective homomorphism $\theta_{i}: R_{i} \rightarrow S_{i}$ which extends to an isomorphism $\bar{\theta}_{i}: \bar{R}_{i} \rightarrow \bar{S}_{i}$. As $S_{i}$ is contained in $\bar{\theta}_{i}\left(\bar{R}_{i}\right)$, we have $\left|S /\left(R+Q_{i}\right)\right|=\left|S_{i} / \theta_{i}\left(R_{i}\right)\right| \leqslant\left|\bar{R}_{i} / R_{i}\right|$.

We have $|S / R|=\prod_{1 \leqslant i \leqslant k}\left|\left(Q_{1} \cdots Q_{i-1}+R\right) /\left(Q_{1} \cdots Q_{i}+R\right)\right|$ with $Q_{1} \cdots Q_{i-1}=S$ for $i=1$ and $Q_{1} \cdots Q_{i}=0$ for $i=k$. For each $i \in\{1, \ldots, k\}$, we have

$$
\begin{aligned}
\left(Q_{1} \cdots Q_{i-1}+R\right) /\left(Q_{1} \cdots Q_{i}+R\right) & =\left(Q_{1} \cdots Q_{i-1}+\left(Q_{1} \cdots Q_{i}+R\right)\right) /\left(Q_{1} \cdots Q_{i}+R\right) \\
& \cong\left(Q_{1} \cdots Q_{i-1}\right) /\left(Q_{1} \cdots Q_{i-1} \cap\left(Q_{1} \cdots Q_{i}+R\right)\right) \\
& =\left(Q_{1} \cdots Q_{i-1}\right) /\left(Q_{1} \cdots Q_{i}+\left(R \cap\left(Q_{1} \cdots Q_{i-1}\right)\right)\right) .
\end{aligned}
$$

Now, let $t$ be an integer such that $(R,+)$ is generated by $t$ elements. Then, for each $i \in\{1, \ldots, k\}$, there are some elements $x_{i, 1}, \ldots, x_{i, t}$ such that $P_{i}=\mathbf{Z} x_{i, 1}+\ldots+\mathbf{Z} x_{i, t}$.

For each $i \in\{1, \ldots, k\}$ each element of $Q_{1} \cdots Q_{i-1}$ can be written as

$$
y=\sum_{1 \leqslant j(1), \ldots, j(i-1) \leqslant t} b_{j(1), \ldots, j(i-1)} x_{1, j(1)} \cdots x_{i-1, j(i-1)}
$$


with $b_{j(1), \ldots, j(i-1)} \in S$ for $j(1), \ldots, j(i-1) \in\{1, \ldots, t\}$ (for $i=1$, we write $y=b \in S$ ). For any integers $j(1), \ldots, j(i-1) \in\{1, \ldots, t\}$, the element $b_{j(1), \ldots, j(i-1)} x_{1, j(1)} \cdots x_{i-1, j(i-1)}$ necessarily belongs to $\left(Q_{1} \cdots Q_{i}\right)+\left(R \cap\left(Q_{1} \cdots Q_{i-1}\right)\right)$ if $b_{j(1), \ldots, j(i-1)}$ belongs to $R+Q_{i}$, since $x_{1, j(1)} \cdots x_{i-1, j(i-1)}$ belongs to $R \cap\left(Q_{1} \cdots Q_{i-1}\right)$. So, for each $i \in\{1, \ldots, k\}$, we have

$$
\left|\left(Q_{1} \cdots Q_{i-1}\right) /\left(\left(Q_{1} \cdots Q_{i}\right)+\left(R \cap\left(Q_{1} \cdots Q_{i-1}\right)\right)\right)\right| \leqslant\left|S /\left(R+Q_{i}\right)\right|^{t^{i-1}} .
$$

Proof of Corollary 2 and Corollary 3: First, we prove Corollaries 2 and 3 for the triples $(A, B, f)$ and $(C, D, g)$ of $(3)$. The proof is essentially the same for the $(n+2)$-tuples $A, B$ of $(2)$, or the pairs $(A, f)$ and $(B, g)$ of (4).

It suffices to show that, for each triple $(A, B, f)$, and for each integer $m \geqslant 2$ such that $m \mathrm{t}(A)=m \mathrm{t}(B)=0$, there exists a $\exists \forall \exists$ sentence $\Phi$ which is satisfied by $(A, B, f)$, and such that the conclusion of [12, Proposition 2.2] is true for any triple $(C, D, g)$ which satisfies $\Phi$ with $m \mathrm{t}(C)=m \mathrm{t}(D)=0$. We consider two finite sequences $\bar{w}$ and $\bar{x}$ which generate $A$ and $B$, and two sequences of variables $\bar{u}, \bar{v}$ such that $\bar{u} \cap \bar{v}=\emptyset,|\bar{u}|=|\bar{w}|$ and $|\bar{v}|=|\bar{x}|$.

For each $i \in\{1, \ldots, n\}$, an element $w \in A$ belongs to $\operatorname{ker}_{i}(f)$ if and only if $(w, \bar{w})$ satisfies the quantifier-free formula $\alpha_{i}(u, \bar{u})$ below:

$$
\mathbb{M}_{u_{1}, \ldots, u_{i-1}, u_{i+1}, \ldots, u_{n} \in \bar{u}} L\left(u_{1}, \ldots, u_{i-1}, u, u_{i+1}, \ldots, u_{n}\right)=0 .
$$

Consequently, $\bar{w}$ satisfies the universal formula $\beta_{i}(\bar{u})$ below:

$$
\left(\forall v_{1} \ldots \forall v_{n}\right)\left(\alpha_{i}\left(v_{i}, \bar{u}\right) \rightarrow L\left(v_{1}, \ldots, v_{n}\right)=0\right) .
$$

The formula $\alpha_{i}(u, \bar{y})$ also defines $\operatorname{ker}_{i}(g)$ in $C$ for each triple $(C, D, g)$ and for each sequence $\bar{y} \subset C$ which satisfies $\beta_{i}$.

For each triple $(C, D, g)$ and for each $i \in\{0, \ldots, n\}$, we write $N_{i}(g)=\bigcap_{1 \leqslant j \leqslant i} \operatorname{ker}_{j}(g)$; we have $N_{0}(g)=C$ and $N_{n}(g)=\operatorname{ker}(g)$. For each $i \in\{1, \ldots, n\}$, we consider the restriction $f_{i}: A^{i-1} \times N_{i-1}(f) \times A^{n-i} \rightarrow B$ of the $n$-linear map $f: A^{n} \rightarrow B$. According to Theorem 2 , there exist a $\forall \exists$ formula $\psi_{i}\left(\bar{u}_{1}^{i}, \ldots, \bar{u}_{n+1}^{i}\right)$ and some sequences $\bar{w}_{1}^{i}, \ldots, \bar{w}_{i-1}^{i} \subset A, \bar{w}_{i}^{i} \subset$ $N_{i-1}(f), \bar{w}_{i+1}^{i}, \ldots, \bar{w}_{n}^{i} \subset A, \bar{w}_{n+1}^{i} \subset B$ such that:

(1) $\left(A, \ldots, A, N_{i-1}(f), A, \ldots, A, B, f_{i}\right)$ satisfies $\psi_{i}\left(\bar{w}_{1}^{i}, \ldots, \bar{w}_{n+1}^{i}\right)$;

(2) for each $(n+2)$-tuple $\left(E_{1}, \ldots, E_{n+1}, h\right)$ such that $m \mathrm{t}\left(E_{1}\right)=\ldots=m \mathrm{t}\left(E_{n+1}\right)$ $=0$, and for any sequences $\bar{y}_{1}, \ldots, \bar{y}_{n+1}$, if $\left(E_{1}, \ldots, E_{n+1}, h\right)$ satisfies $\psi_{i}\left(\bar{y}_{1}, \ldots, \bar{y}_{n+1}\right)$, then $E_{i}$ is generated by $\bar{y}_{i}$ and $\operatorname{ker}_{i}(h)$.

For each $i \in\{1, \ldots, n\}$, there exists some sequences of terms $\bar{\tau}_{1}^{i}(\bar{u}), \ldots, \bar{\tau}_{n}^{i}(\bar{u}), \bar{\tau}_{n+1}^{i}(\bar{v})$ such that $\bar{w}_{1}^{i}=\bar{\tau}_{1}^{i}(\bar{w}), \ldots, \bar{w}_{n}^{i}=\bar{\tau}_{n}^{i}(\bar{w}), \bar{w}_{n+1}^{i}=\bar{\tau}_{n+1}^{i}(\bar{x})$. We consider the $\forall \exists$ formula $\varphi(\bar{u}, \bar{v})=\beta_{1} \wedge \cdots \wedge \beta_{n} \wedge \varphi_{1} \wedge \cdots \wedge \varphi_{n}$ where, for each $i \in\{1, \ldots, n\}, \varphi_{i}(\bar{u}, \bar{v})$ is obtained from $\psi_{i}\left(\bar{u}_{1}^{i}, \ldots, \vec{u}_{n+1}^{i}\right)$ by replacing successively: 
- each variable in $\bar{u}_{1}^{i}, \ldots, \bar{u}_{n+1}^{i}$ by the corresponding term in $\bar{u}$ or $\bar{v}$;

- each subformula $\left(\exists v_{i}\right) \theta$, where $v_{i}$ is a variable which represents an element of the $i$-th group, by $\left(\exists v_{i}\right)\left(\alpha_{1}\left(v_{i}, \bar{u}\right) \wedge \ldots \wedge \alpha_{i-1}\left(v_{i}, \bar{u}\right) \wedge \theta\right)$;

- each subformula $\left(\forall v_{i}\right) \theta$, where $v_{i}$ is a variable which represents an element of the $i$-th group, by $\left(\forall v_{i}\right)\left[\left(\alpha_{1}\left(v_{i}, \bar{u}\right) \wedge \ldots \wedge \alpha_{i-1}\left(v_{i}, \bar{u}\right)\right) \rightarrow \theta\right]$.

The triple $(A, B, f)$ satisfies $\varphi(\bar{w}, \bar{x})$. For each finitely generated triple $(C, D, g)$ such that $m \mathrm{t}(C)=m \mathrm{t}(D)=0$, and for any sequences $\bar{y} \subset C$ and $\bar{z} \subset D$, if $(C, D, g)$ satisfies $\varphi(\bar{y}, \bar{z})$, then $C$ is generated by $\bar{y}$ and $\operatorname{ker}(g)$ since, for each $i \in\{1, \ldots, n\}, N_{i-1}(g)$ is generated by $\bar{\tau}_{i}^{i}(\bar{y})$ and $N_{i}(g)$.

Then, we define $\Sigma(\bar{u}, \bar{v})$ as in the proof of [12, Proposition 2.2], with $m$ instead of $r$ and $s$, and we consider the $\exists \forall \exists$ sentence $\Phi=(\exists \bar{u})(\exists \bar{v}) \Sigma$.

Now, we prove Corollaries 2 and 3 for finitely generated finite-by-nilpotent groups. It suffices to show that, for each finitely generated finite-by-nilpotent group $G$ and for any integers $c, m \geqslant 1$, if $\Gamma_{c+1}(G)$ is finite and $\mathrm{t}\left(\Gamma_{i}(G) / \Gamma_{i+1}(G)\right)^{m}=1$ for $1 \leqslant i \leqslant c$, then $G$ satisfies a $\exists \forall \exists$ sentence $\Phi$ such that the conclusion of [11, Proposition 1] is true for any finitely generated finite-by-nilpotent group $H$ which satisfies $\Phi$ with $\mathrm{t}\left(\Gamma_{i}(H) / \Gamma_{i+1}(H)\right)^{m}=1$ for $1 \leqslant i \leqslant c$.

We see from the proof of $[11$, Lemma 1.1] that, for each integer $i \geqslant 1$, there exist a $\forall \exists$ sentence $\theta_{i}$ which is true in $G$ and an existential formula $\gamma_{i}$ which defines $\Gamma_{i}(H)$ in $H$ for any group $H$ which satisfies $\theta_{i}$. We consider the groups $A_{i}=\Gamma_{i}(G) / \Gamma_{i+1}(G)$ and the quadruples $M_{i}=\left(A_{1}, A_{i}, A_{i+1}, g_{i}\right)$, where $g_{i}$ is the bilinear map induced by the map $(x, y) \rightarrow[x, y]$.

According to Theorem 2, for each $i \in\{1, \ldots, c-1\}$, there exist some disjoint sequences of variables $\bar{u}_{1, i}, \bar{u}_{2, i}, \bar{u}_{3, i}$, some sequences $\bar{x}_{1, i}^{*} \subset A_{1}, \bar{x}_{2, i}^{*} \subset A_{i}, \bar{x}_{3, i}^{*} \subset A_{i+1}$, and a conjunction of $\sharp$ formulas $\varphi_{i}^{*}\left(\bar{u}_{1, i}, \bar{u}_{2, i}, \bar{u}_{3, i}\right)$ such that:

(1) $M_{i}$ satisfies $\varphi_{i}^{*}\left(\bar{x}_{1, i}^{*}, \bar{x}_{2, i}^{*}, \bar{x}_{3, i}^{*}\right)$;

(2) For each quadruple $N=\left(N_{1}, N_{2}, N_{3}, h\right)$ such that $\mathrm{t}\left(N_{1}\right)^{m}=\mathrm{t}\left(N_{2}\right)^{m}=$ $\mathrm{t}\left(N_{3}\right)^{m}=1$, and for any sequences $\bar{y}_{1} \subset N_{1}, \bar{y}_{2} \subset N_{2}, \bar{y}_{3} \subset N_{3}$, if $N$ satisfies $\varphi_{i}^{*}\left(\bar{y}_{1}, \bar{y}_{2}, \bar{y}_{3}\right)$, then $N_{1}=\left\langle\bar{y}_{1}, \operatorname{ker}_{1}(h)\right\rangle$ and $N_{2}=\left\langle\bar{y}_{2}, \operatorname{ker}_{2}(h)\right\rangle$.

For each $i \in\{1, \ldots, c-1\}$, we consider the $\forall \exists$ formula $\varphi_{i}\left(\bar{u}_{1, i}, \bar{u}_{2, i}, \bar{u}_{3, i}\right)$ in the language of groups which is obtained from $\varphi_{i}^{*}\left(\bar{u}_{1, i}, \bar{u}_{2, i}, \bar{u}_{3, i}\right)$ by doing successively the substitutions below:

(a) Replace each atomic subformula $\tau_{1}\left(\bar{w}_{1}\right)=1, \tau_{2}\left(\bar{w}_{2}\right)=1$ or $\tau_{3}\left(\bar{w}_{3}\right)$ $\prod_{1 \leqslant i \leqslant n} L\left(\tau_{1, i}\left(\bar{w}_{1}\right), \tau_{2, i}\left(\bar{w}_{2}\right)\right)=1$ by the corresponding positive existential formula $\gamma_{2}\left(\tau_{1}\left(\bar{w}_{1}\right)\right), \gamma_{i+1}\left(\tau_{2}\left(\bar{w}_{2}\right)\right)$ or $\gamma_{i+2}\left(\tau_{3}\left(\bar{w}_{3}\right) \prod_{1 \leqslant i \leqslant n}\left[\tau_{1, i}\left(\bar{w}_{1}\right), \tau_{2, i}\left(\bar{w}_{2}\right)\right]\right)$. This step only creates existential quantifiers inside the positive existential part of each $\sharp$ subformula of $\varphi_{i}^{*}$.

(b) Substitute the existential quantifiers as follows: $\left(\exists w_{1}\right) \theta\left(w_{1}, \bar{w}\right)$ remains as it 
is, but $\left(\exists w_{2}\right) \theta\left(w_{2}, \bar{w}\right)$ and $\left(\exists w_{3}\right) \theta\left(w_{3}, \bar{w}\right)$ respectively become $\left(\exists w_{2}\right)\left(\gamma_{i}\left(w_{2}\right) \wedge\right.$ $\left.\theta\left(w_{2}, \bar{w}\right)\right)$ and $\left(\exists w_{3}\right)\left(\gamma_{i+1}\left(w_{3}\right) \wedge \theta\left(w_{3}, \bar{w}\right)\right)$. This step only creates existential quantifiers.

(c) Substitute the universal quantifiers as follows: $\left(\forall w_{1}\right) \theta\left(w_{1}, \bar{w}\right)$ remains as it is, but $\left(\forall w_{2}\right) \theta\left(w_{2}, \bar{w}\right)$ and $\left(\forall w_{3}\right) \theta\left(w_{3}, \bar{w}\right)$ respectively become $\left(\forall w_{2}\right)\left(\gamma_{i}\left(w_{2}\right)\right.$ $\left.\rightarrow \theta\left(w_{2}, \bar{w}\right)\right)$ and $\left(\forall w_{3}\right)\left(\gamma_{i+1}\left(w_{3}\right) \rightarrow \theta\left(w_{3}, \bar{w}\right)\right)$. This step only creates universal quantifiers, since the existential quantifiers are introduced in $\gamma_{i}\left(w_{2}\right)$ and $\gamma_{i+1}\left(w_{3}\right)$, which appear in a negative form.

The group $G$ satisfies $\varphi_{i}\left(\bar{x}_{1, i}, \bar{x}_{2, i}, \bar{x}_{3, i}\right)$ for any representatives $\bar{x}_{1, i}, \bar{x}_{2, i}, \bar{x}_{3, i}$ of $\bar{x}_{1, i}^{*}, \bar{x}_{2, i}^{*}$, $\bar{x}_{3, i}^{*}$ in $G, \Gamma_{i}(G), \Gamma_{i+1}(G)$. For each finitely generated finite-by-nilpotent group $H$ and for any sequences $\bar{y}_{1}, \bar{y}_{2}, \bar{y}_{3}$, if $H$ satisfies $\theta_{1} \wedge \ldots \wedge \theta_{i+2} \wedge \varphi_{i}\left(\bar{y}_{1}, \bar{y}_{2}, \bar{y}_{3}\right)$, and if $\mathrm{t}\left(\Gamma_{j}(H) / \Gamma_{j+1}(H)\right)^{m}$ $=1$ for $j=1, i, i+1$, then we have $H=\left\langle\bar{y}_{1},\left\{y \in H \mid\left[y, \Gamma_{i}(H)\right] \subset \Gamma_{i+2}(H)\right\}\right\rangle$ and $\Gamma_{i}(H)=\left\langle\bar{y}_{2},\left\{y \in \Gamma_{i}(H) \mid[H, y] \subset \Gamma_{i+2}(H)\right\}\right\rangle$. It follows $\Gamma_{i+1}(H)=\left\langle\left[\bar{y}_{1}, \bar{y}_{2}\right], \Gamma_{i+2}(H)\right\rangle$.

Now, we consider a finite sequence $\bar{x}$ which generates $G$, a sequence of variables $\bar{u}$ with $|\bar{u}|=|\bar{x}|$, and:

(1) some terms $\rho_{1}(\bar{u}), \ldots, \rho_{p}(\bar{u})$ such that $\left\langle\bar{x} ; \rho_{1}(\bar{x}), \ldots, \rho_{p}(\bar{x})\right\rangle$ is a presentation of $G$ on $\bar{x}$, and the formula $\rho_{1}(\bar{u})=1 \wedge \ldots \wedge \rho_{p}(\bar{u})=1$;

(2) the integer $q=\left|\Gamma_{c+1}(G)\right|$, some terms $\sigma_{1}(\bar{u}), \ldots, \sigma_{q}(\bar{u})$ such that $\Gamma_{c+1}(G)=$ $\left\{\sigma_{1}(\bar{x}), \ldots, \sigma_{q}(\bar{x})\right\}$, and the formula

$$
\left.\left[\mathbb{X}_{1 \leqslant i<j \leqslant q}\right\urcorner\left(\sigma_{i}(\bar{u})=\sigma_{j}(\bar{u})\right)\right] \wedge(\forall v)\left[\gamma_{c+1}(v) \rightarrow\left(\mathbb{W}_{1 \leqslant i \leqslant q} v=\sigma_{i}(\bar{u})\right)\right] ;
$$

(3) for $1 \leqslant i \leqslant c-1$, some sequences of terms $\bar{\xi}_{1, i}(\bar{u}), \bar{\xi}_{2, i}(\bar{u}), \bar{\xi}_{3, i}(\bar{u})$ such that $\bar{\xi}_{1, i}(\bar{x}), \bar{\xi}_{2, i}(\bar{x}), \bar{\xi}_{3, i}(\bar{x})$ are representatives of $\bar{x}_{1, i}^{*}, \bar{x}_{2, i}^{*}, \bar{x}_{3, i}^{*}$ in $G, \Gamma_{i}(G), \Gamma_{i+1}(G)$, and the formula $\varphi_{i}^{\prime}(\bar{u})$ which is obtained from $\varphi_{i}\left(\bar{u}_{1, i}, \bar{u}_{2, i}, \bar{u}_{3, i}\right)$ by replacing $\bar{u}_{1, i}, \bar{u}_{2, i}, \bar{u}_{3, i}$ with $\bar{\xi}_{1, i}(\bar{u}), \bar{\xi}_{2, i}(\bar{u}), \bar{\xi}_{3, i}(\bar{u}) ;$

(4) some terms $\tau_{1}(\bar{u}), \ldots, \tau_{r}(\bar{u})$ such that $\tau_{1}(\bar{x}), \ldots, \tau_{r}(\bar{x})$ are representatives in $G$ of the elements of $G /\left\langle G^{m}, \Gamma_{2}(G)\right\rangle$, and the formula

$$
\begin{aligned}
&(\forall v)\left(\exists w_{1}\right)\left(\exists w_{2}\right)\left[\gamma_{2}\left(w_{2}\right) \wedge\left(W_{1 \leqslant i \leqslant r} v=\tau_{i}(\bar{u}) w_{1}^{m} w_{2}\right)\right] \\
& \wedge\left[\mathbb{M}_{1 \leqslant i<j \leqslant r}\left(\forall w_{1}\right)\left(\forall w_{2}\right)\left[\gamma_{2}\left(w_{2}\right) \rightarrow \neg\left(\tau_{i}(\bar{u})=\tau_{j}(\bar{u}) w_{1}^{m} w_{2}\right)\right]\right]
\end{aligned}
$$

(5) the sentence $\theta=\theta_{1} \wedge \ldots \wedge \theta_{c+1}$;

(6) a prime number $\pi$ which does not divide $m$; for $1 \leqslant i \leqslant c$, the integer $s(i)$ 
such that $\left|\Gamma_{i}(G) /\left\langle\Gamma_{i}(G)^{\pi m}, \Gamma_{i+1}(G)\right\rangle\right|=s(i)$, and the sentence

$$
\begin{aligned}
\left(\exists v_{1} \ldots \exists v_{s(i)}\right)\left\{\gamma_{i}\left(v_{1}\right) \wedge \ldots \wedge\right. & \gamma_{i}\left(v_{s(i)}\right) \wedge(\forall v)\left[\gamma _ { i } ( v ) \rightarrow ( \exists w _ { 1 } ) ( \exists w _ { 2 } ) \left[\gamma_{i}\left(w_{1}\right)\right.\right. \\
& \left.\left.\wedge \gamma_{i+1}\left(w_{2}\right) \wedge\left(W_{1 \leqslant j \leqslant s(i)} v=v_{j} w_{1}^{\pi m} w_{2}\right)\right]\right] \\
& \wedge\left[\bigwedge _ { 1 \leqslant j < k \leqslant s ( i ) } ( \forall w _ { 1 } ) ( \forall w _ { 2 } ) \left[\left(\gamma_{i}\left(w_{1}\right)\right.\right.\right. \\
& \left.\left.\left.\left.\wedge \gamma_{i+1}\left(w_{2}\right)\right) \rightarrow \neg\left(v_{j}=v_{k} w_{1}^{\pi m} w_{2}\right)\right]\right]\right\} .
\end{aligned}
$$

The conjunction $\varphi(\bar{u})$ of the formulas in (1), (2), (3), (4) is $\forall \exists$, and the conjunction $\psi$ of the sentences in (5), (6) is $\exists \forall \exists$. The $\exists \forall \exists$ sentence $\Phi=\psi \wedge(\exists \bar{u}) \varphi$ is satisfied by $\bar{x}$ in $G$.

Now, let us consider a finitely generated finite-by-nilpotent group $H$ which satisfies $\Phi$, with $\mathrm{t}\left(\Gamma_{i}(H) / \Gamma_{i+1}(H)\right)^{m}=1$ for $1 \leqslant i \leqslant c$, and a sequence $\bar{y} \subset H$ which satisfies $\varphi$.

According to (5), $\gamma_{i}$ defines $\Gamma_{i}(H)$ in $H$ for $1 \leqslant i \leqslant c+1$. For $1 \leqslant i \leqslant c$, the finitely generated Abelian groups $A_{i}=\Gamma_{i}(G) / \Gamma_{i+1}(G)$ and $B_{i}=\Gamma_{i}(H) / \Gamma_{i+1}(H)$ are isomorphic since they satisfy $\left|A_{i} / A_{i}^{\pi m}\right|=\left|B_{i} / B_{i}^{\pi m}\right|$ by $(6)$, and $\mathrm{t}\left(A_{i}\right)^{m}=\mathrm{t}\left(B_{i}\right)^{m}=1$.

According to (1), the map $\bar{x} \rightarrow \bar{y}$ extends to a homomorphism $f: G \rightarrow H$. By (2), $f$ induces an isomorphism from $\Gamma_{c+1}(G)$ to $\Gamma_{c+1}(H)$. For $2 \leqslant i \leqslant c$, we infer from (3) and the properties of $\varphi_{i-1}$ that $\left.\Gamma_{i}(H)=\left\langle\bar{\xi}_{1, i-1}(\bar{y}), \bar{\xi}_{2, i-1}(\bar{y})\right], \Gamma_{i+1}(H)\right\rangle$; consequently, $f$ induces a surjective homomorphism, and therefore an isomorphism, from $A_{i}$ to $B_{i}$. It follows that $f$ induces an isomorphism from $\Gamma_{2}(G)$ to $\Gamma_{2}(H)$.

By (4), $f$ induces an isomorphism from $A_{1} / A_{1}^{m}=G /\left\langle G^{m}, \Gamma_{2}(G)\right\rangle$ to $B_{1} / B_{1}^{m}=$ $H /\left\langle H^{m}, \Gamma_{2}(H)\right\rangle$. As $A_{1}$ and $B_{1}$ are isomorphic, and $\mathrm{t}\left(A_{1}\right)^{m}=\mathrm{t}\left(B_{1}\right)^{m}=1$, it follows that $f$ induces an injective homomorphism $\bar{f}: A_{1} \rightarrow B_{1}$ with $\left|B_{1} / \bar{f}\left(A_{1}\right)\right|$ prime to $m$ (see [10, p.66]). In particular, $f$ induces an isomorphism from $\mathrm{t}\left(A_{1}\right)=\Delta(G) / \Gamma_{2}(G)$ to $\mathrm{t}\left(B_{1}\right)=\Delta(H) / \Gamma_{2}(H)$.

Consequently, $f$ satisfies the conclusion of $[11$, Proposition 1]: $f$ is an injective homomorphism from $G$ to $H$ with $f(\Delta(G))=\Delta(H)$ and $|H / f(G)|$ prime to $m$.

\section{REFERENCES}

[1] S. Burris, 'Bounded Boolean powers and $\equiv_{n}$ ', Algebra Universalis 8 (1978), 137-138.

[2] C.C. Chang and H.J. Keisler, Model theory, Studies in Logic 73 (North-Holland, Amsterdam, 1973).

[3] S. Lang, Algebra (Addison-Wesley, Reading, MA, 1978).

[4] A.G. Myasnikov, Ninth All-union Symposium on Group Theory (Moscow State Univ., Moscow, 1984), pp. 46-47.

[5] A.G. Myasnikov, 'Definable Invariants of bilinear mappings', Siberian Math. J. 31 (1990), 89-99.

[6] A.G. Myasnikov, 'The theory of models of bilinear mappings', Siberian Math. J. 31 (1990), 439-451. 
[7] F. Oger, 'Equivalence élémentaire entre groupes finis-par-abéliens de type fini', Comment Math. Helv. 57 (1982), 469-480.

[8] F. Oger, 'Des groupes nilpotents de classe 2 sans torsion de type fini peuvent ne pas être élémentairement équivalents', C.R. Acad. Sci. Paris Sér. I Math. 294 (1982), 1-4.

[9] F. Oger, 'Elementary equivalence and profinite completions: a characterization of finitely generated abelian-by-finite groups', Proc. Amer. Math. Soc. 103 (1988), 1041-1048.

[10] F. Oger, 'Elementary equivalence and genus of finitely generated nilpotent groups', Bull. Austral. Math. Soc. 37 (1988), 61-68.

[11] F. Oger, 'Cancellation and elementary equivalence of finitely generated finite-by-nilpotent groups', J. London Math. Soc. 44 (1991,), 173-183.

[12] F. Oger, 'Isomorphism and elementary equivalence of multilinear maps', Linear and Multilinear Algebra 36 (1994), 151-174.

[13] F. Oger, 'Elementary equivalence for abelian-by-finite and nilpotent groups' (to appear).

[14] M. Prest, Model theory and modules, London Math. Soc. Lecture Notes Series 130 (Cambridge University Press, Cambridge, 1988).

[15] D. Raphael, 'Commensurability and elementary equivalence of polycyclic groups', Bull. Austral. Math. Soc. 53 (1996), 425-439.

[16] D. Raphael, Comparaison de propriétés modèle-théoriques et de propriétés algébriques des groupes polycycliques-par-finis, Doctoral dissertation (University Paris VII, Paris, 1996).

[17] R.B. Warfield, Jr., Nilpotent groups, Lecture Notes in Math. 513 (Springer-Verlag, Berlin, 1976).

Equipe de Logique Mathematique

Université Paris VII - C.N.R.S.

2 place Jussieu, case 7012

75251 Paris Cédex 05

France

e-mail: oger@logique.jussieu.fr 